\title{
Lepton Events with Missing Transverse Momentum and Single Top (FCNC) at HERA
}

\author{
Claude Vallée* $\dagger$ \\ Centre de Physique des Particules de Marseille, \\ 163 Avenue de Luminy, case 907, \\ F-13288 Marseille cedex 9, France \\ E-mail: ivallee@cppm.in2p3. fri
}

\begin{abstract}
The latest results of HERA searches for lepton events with missing transverse momentum are presented. They are based on the full HERA I data sets, corresponding to integrated luminosities of $130 \mathrm{pb}^{-1}$ (ZEUS) and $116 \mathrm{pb}^{-1}$ (H1). At low hadronic $P_{T}$, a good agreement is found with the Standard Model prediction, dominated by $W$ production. At high hadronic $P_{T}, \mathrm{H} 1$ observes more events than expected. Several of them are kinematically compatible with a single top quark decay. Complementary searches by both experiments for a top signal in the hadronic channel show no excess compared to Standard Model predictions. Limits on a possible anomalous FCNC coupling of the top quark to the u quark and the photon are derived.
\end{abstract}

\section{Lepton events with missing transverse momentum}

\subsection{Topologies and selection strategies}

The events are required to contain at least one isolated lepton (electron or muon) with a high transverse momentum $P_{T}^{l}$, together with missing transverse momentum $P_{T}^{\text {miss }}$. They may also contain a hadronic system $X$ with transverse momentum $P_{T}^{X}$. The H1 selection [i] is optimised for $W$-like topologies, whereas ZEUS $[2 \overline{2}$ ] performed both an inclusive analysis dominated by non- $W$ processes, and a search tuned for $W$ 's at high- $P_{T}^{X}$. The analysed data sets correspond to a factor 3 increase in integrated luminosity compared to published

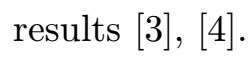

${ }^{*}$ Speaker.

${ }^{\dagger}$ On behalf of the H1 and ZEUS Collaborations. 


\subsection{Results}

The observed event yields are summarized in Table ${ }_{-i}^{1}$ Figures 1 and 2 show the distributions of the $\mathrm{H} 1$ candidates in the lepton-neutrino transverse mass $M_{T}\left(P_{T}^{\text {miss }}\right.$ being attributed to a hypothetical neutrino), and in $P_{T}^{X}$.

\begin{tabular}{|c|c|c|}
\hline & H1 preliminary & ZEUS preliminary \\
\hline$P_{T}^{X}$ range & Data/SM & Data/SM \\
\hline & 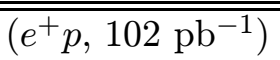 & 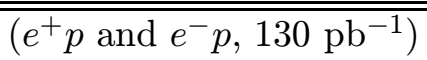 \\
\hline$P_{T}^{X}>0 \mathrm{GeV}$ & $18 / 10.5 \pm 2.5$ & $17 / 16.4 \pm 1.7$ \\
\hline$P_{T}^{X}>25 \mathrm{GeV}$ & $10 / 2.8 \pm 0.7$ & $2 / 2.4 \pm 0.2$ \\
\hline$P_{T}^{X}>40 \mathrm{GeV}$ & $6 / 1.0 \pm 0.3$ & $0 / 1.0 \pm 0.1$ \\
\hline & $\left(e^{-} p, 14 \mathrm{pb}^{-1}\right)$ & \\
\hline$P_{T}^{X}>0 \mathrm{GeV}$ & $0 / 1.8 \pm 0.4$ & \\
\hline
\end{tabular}

Table 1: Lepton event yields compared to SM predictions. The SM expectations are dominated by $W$-production except for the ZEUS inclusive analysis quoted in brackets.

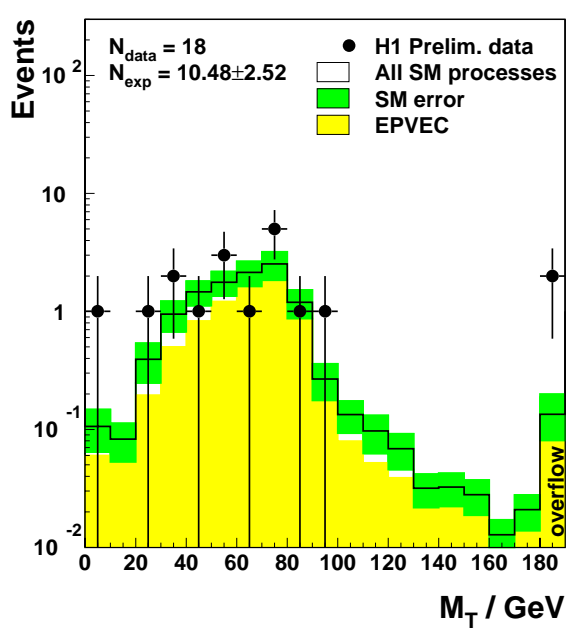

Figure 1: Lepton-neutrino transverse mass distribution of the $\mathrm{H} 1$ lepton events, compared to the SM prediction $\left(e^{+} p\right.$ data $)$.

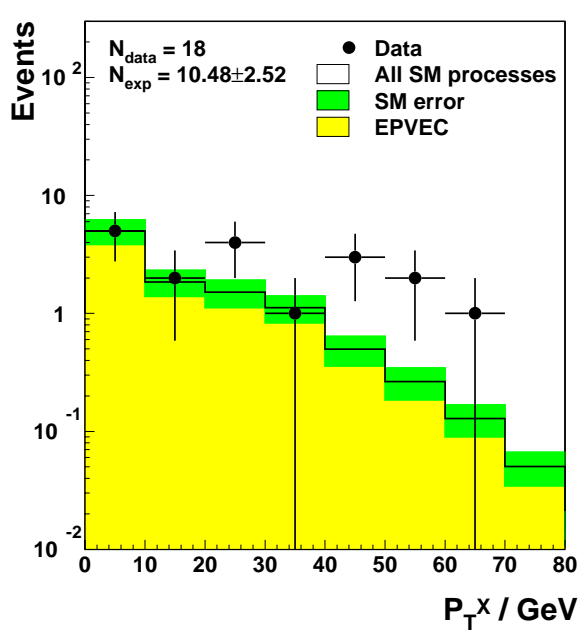

Figure 2: Hadronic transverse momentum distribution of the $\mathrm{H} 1$ lepton events, compared to the SM prediction $\left(e^{+} p\right.$ data).

The $M_{T}$ variable follows a Jacobian peak distribution around the $W$ boson mass. This favours the interpretation of most events as real $W$ production. At low $P_{T}^{X}$, the measured rates agree with the Standard Model (SM) expectation. At high $P_{T}^{X}$, ZEUS observes agreement with the SM, whereas $\mathrm{H} 1$ finds more events than expected. The expectations

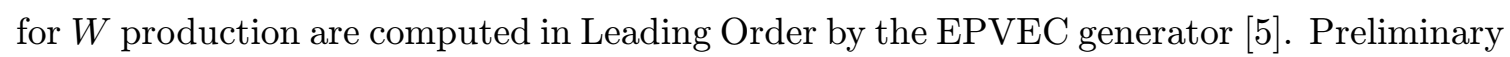

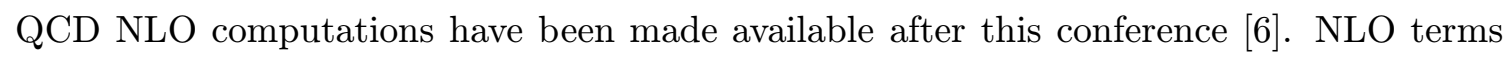
are found to change the $W$ yield by less than $10 \%$ at high $P_{T}^{X}$. 


\section{Single top searches}

A possible interpretation of the high $P_{T}^{X} \mathrm{H} 1$ events could be top production, with the hadronic system $X$ corresponding to the secondary $b$-quark of the top decay. The SM

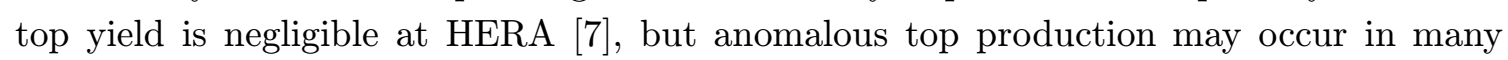
extensions of the SM [is . This process can be described in a model independent way by an effective lagrangian [i] involving Flavour Changing Neutral Currents (FCNC's) quantified by anomalous couplings of the top to other quarks and neutral gauge bosons. At HERA the dominant contribution is expected from an anomalous coupling $\kappa_{t u \gamma}$ to the u-quark and the photon. This, together with the observed lepton events, motivated dedicated top searches

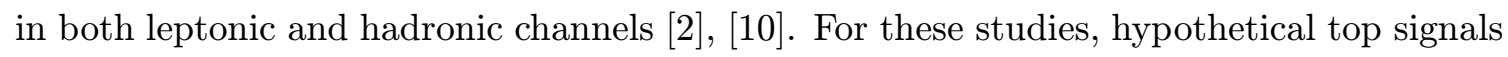
have been simulated using either the effective lagrangian of [ij] (H1) or an excited fermion generator at the top mass (ZEUS).

\subsection{Leptonic channel}

The selections of Section 1 were further tuned for top searches, adding a threshold in $P_{T}^{X}$ of $40 \mathrm{GeV}$ (ZEUS) and $35 \mathrm{GeV}$ or $25 \mathrm{GeV}$ (H1, depending on the hadronic jet angular range). $\mathrm{H} 1$ also requires a positive lepton charge and $M_{T}$ higher than $10 \mathrm{GeV}$. With these cuts $\mathrm{H} 1$ observes 5 events with $1.8 \pm 0.5$ expected, whereas ZEUS sees no event, with 1.0 expected.

Within the $W$ interpretation, the lepton-neutrino-X mass $M_{l \nu X}$ of the $\mathrm{H} 1$ candidates can be computed after reconstructing the neutrino from the $W$ mass constraint. For each event two mass solutions arise. For three candidates only one solution fits the overall kinematic constraints, whereas for the other two candidates both solutions are acceptable. The resulting $M_{l \nu X}$ distributions are shown in Figure $\overline{\underline{3}}$.
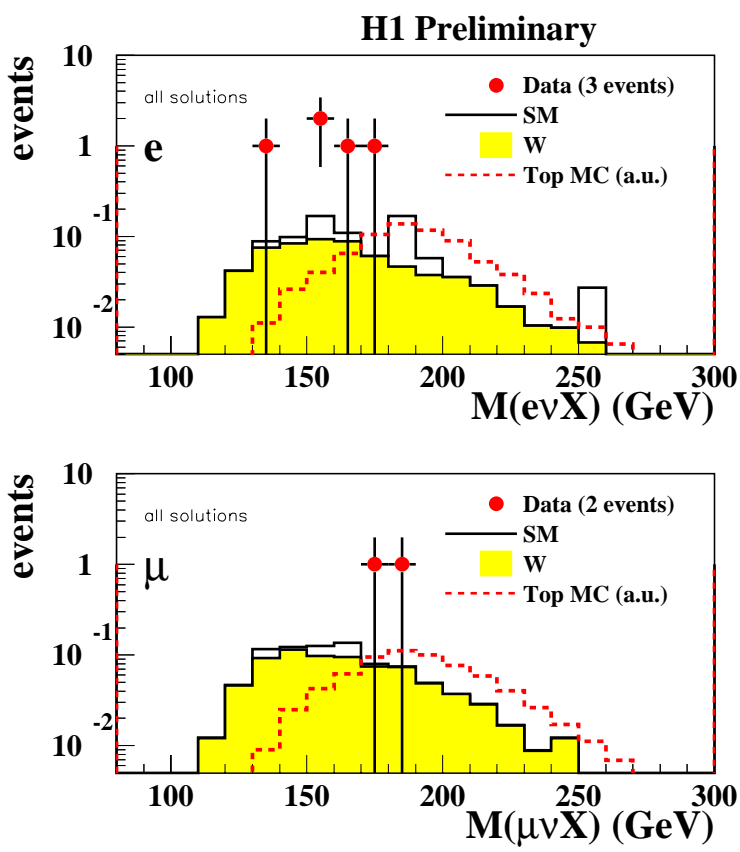

Figure 3: $M_{l \nu X}$ distributions of the H1 top candidates, compared to expectations from SM $W$ production and a hypothetical top signal.

$M_{l \nu X}$ is compatible with the top mass for several events.

\subsection{Hadronic channel}

The hadronic decay of a top quark produces one high- $P_{T}$ jet associated with the secondary $b$-quark and two high- $P_{T}$ jets from the secondary $W$ decay. The experimental selection 


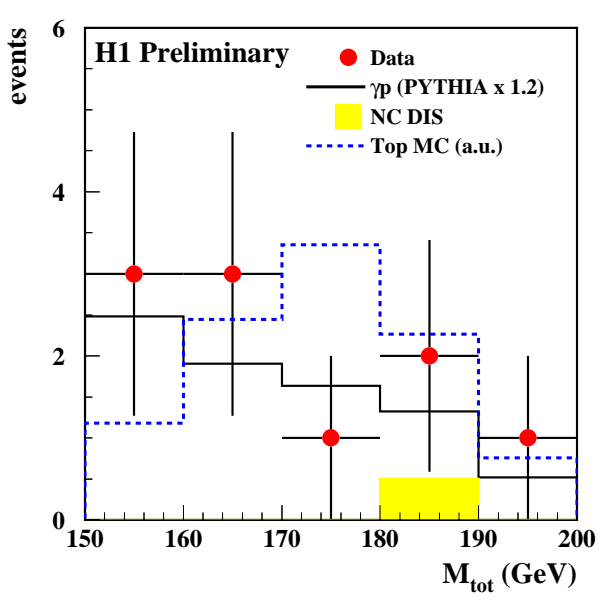

Figure 4: 3-jets mass distributions of the H1 top candidates in the hadronic channel, compared to expectations of SM QCD background and a hypothetical top signal.

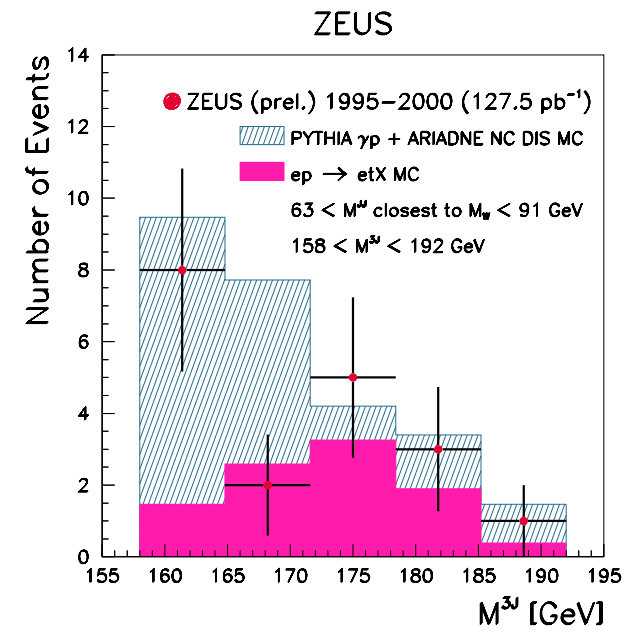

Figure 5: 3-jets mass distributions of the ZEUS top candidates in the hadronic channel, compared to expectations of SM QCD background and a hypothetical top signal.

therefore requires three jets with $P_{T}$ 's above 40, 25 and $14 \mathrm{GeV}$ (ZEUS) or 25, 15 and $10 \mathrm{GeV}$ (H1). H1, in addition, asks for a total transverse energy above $120 \mathrm{GeV}$. Both experiments further reduce the QCD photoproduction background by requiring the 3jets mass and one of the 2-jets masses to be compatible with the top mass and the $W$ mass, respectively. The searches were performed on data sets corresponding to integrated luminosities of $128 \mathrm{pb}^{-1}$ (ZEUS) and $37 \mathrm{pb}^{-1}$ (H1).

After applying the above selections the observed event yields are 10 for $8.3_{-1.9}^{+4.2}(\exp )$ \pm 4.2 (theory) expected (H1) and 19 for 20.0 expected (ZEUS). In order to reduce higherorder QCD uncertainties, the SM predictions are obtained by normalising the simulated rates to those observed in the low-mass domain. The 3-jets mass distributions of the $\mathrm{H} 1$ and ZEUS candidates are shown in Figures 4 and 5, respectively.

Both experiments find no excess compared to SM predictions. This does not favour the interpretation of the $\mathrm{H} 1$ high- $P_{T}^{X}$ lepton events as top production. It, however, does not rule it out since $95 \%$ C.L. upper limits on the top cross-section derived from the hadronic channel only would correspond to more than 3 top events expected in the H1 lepton event sample.

\subsection{Limits on anomalous couplings}

Combining the leptonic and hadronic channels, upper limits on top production crosssections were set. Using the effective lagrangian of [iq $]$, they were converted into $95 \%$ C.L. upper bounds on the anomalous coupling $\kappa_{t u \gamma}$ of 0.19 (ZEUS) and 0.305 (H1). The 
ZEUS limit is more stringent than the H1 limit because of the higher integrated luminosity analysed in the hadronic channel and the absence of top candidates in the leptonic channel. The ZEUS 95\% C.L. upper limit on the top cross-section corresponds to about 2 top events expected in the $\mathrm{H} 1$ lepton event sample.

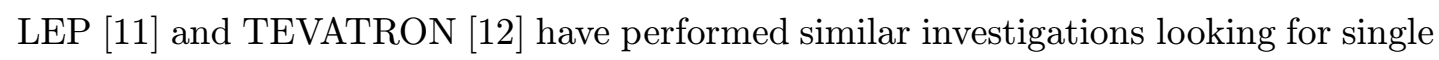
top production and rare top decays, respectively. They are sensitive to anomalous couplings of the top to the $u$ - and $c$-quarks through both the photon and the $Z$ boson. Their results are compared to HERA limits in Figure of anomalous top physics.

\section{Outlook}

The HERA collider is now starting a new phase of operation at higher luminosity. The goal is to accumulate, in the next five years, ten times more data than presently available. This will clarify the origin of the high- $P_{T}^{X}$ lepton events observed by H1. Enhanced $b$-tagging capabilities of the new $\mathrm{H} 1$ and ZEUS vertex detectors will also improve the signatures for unexpected new phenomena such as anomalous top production.

\section{Acknowledgments}

I am indebted to all my ZEUS and H1 Colleagues for their outstanding work in producing these results.

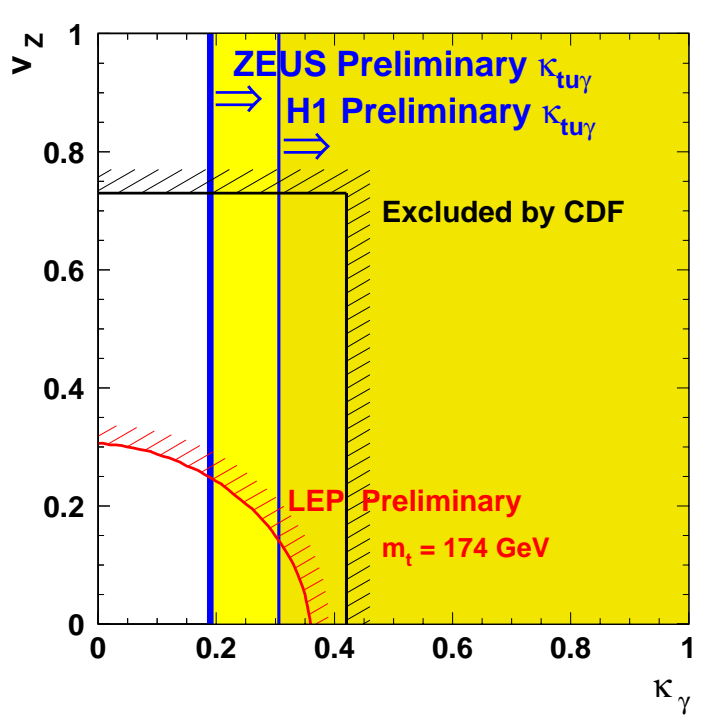

Figure 6: Present limits of LEP, TEVATRON and HERA on anomalous couplings of the top quark to other quarks and neutral gauge bosons.

\section{References}

[1] H1 Collaboration, contributed paper 802 to this Conference.

[2] ZEUS Collaboration, contributed paper 650 to this Conference.

[3] C.Adloff et al., (H1 Collaboration), 'Eur. Physs. J.

[4] J.Breitweg et al., (ZEUS Collaboration), Phys. Lett. B $\mathbf{4} 7 \overline{1}-(2000) 41 \overline{1}_{1}^{\prime}$

[5] U.Baur, J.A.M.Vermaseren and D.Zeppenfeld, 'Nucucl. Phys. B B 375_(1992) 3.

[6] C.Schwanenberger, DESY Theory Workshop, October 11th, 2001, Hamburg, Germany.

[7] S.Moretti and K.Odagiri, 
[8] D.Atwood, L.Reina and A.Soni, 'Phys. Rev. D $\mathbf{5 3}(1996) 1199 ;$

G.M.Divitiis, R.Petronzio and L.Silvestrini, 'Nucl. Phys. B $\mathbf{5 0 4}$ (1997)_45r

T.Han, R.D.Peccei and X.Zhang, 'Nucl. Phys. B

H.Fritzsch and D.Holtmannspötter, 'Phys. Lett. B $45 \overline{4}(19 \overline{9})$ 1 1999 .

[9] T.Han and J.L.Hewett, 'P

[10] H1 Collaboration, contributed paper 824 to this Conference.

[11] M.Pimenta (LEP Collaborations), these proceedings.

[12] F.Abe et al., (CDF Collaboration), PPhys. Rev. Lett. 80 (1998) 2525. 